\title{
Providing Assistance for Orienting 3D Objects Using Monocular Eyewear
}

\author{
Mengu Sukan Carmine Elvezio Steven Feiner \\ Dept. of Computer Science, Columbia University \\ New York, NY, 10027 USA \\ \{mengu, carmine, feiner\}@cs.columbia.edu
}

\author{
Barbara Tversky \\ Dept. of Human Dev., Teachers College \\ New York, NY, 10027 USA \\ btversky@stanford.edu
}
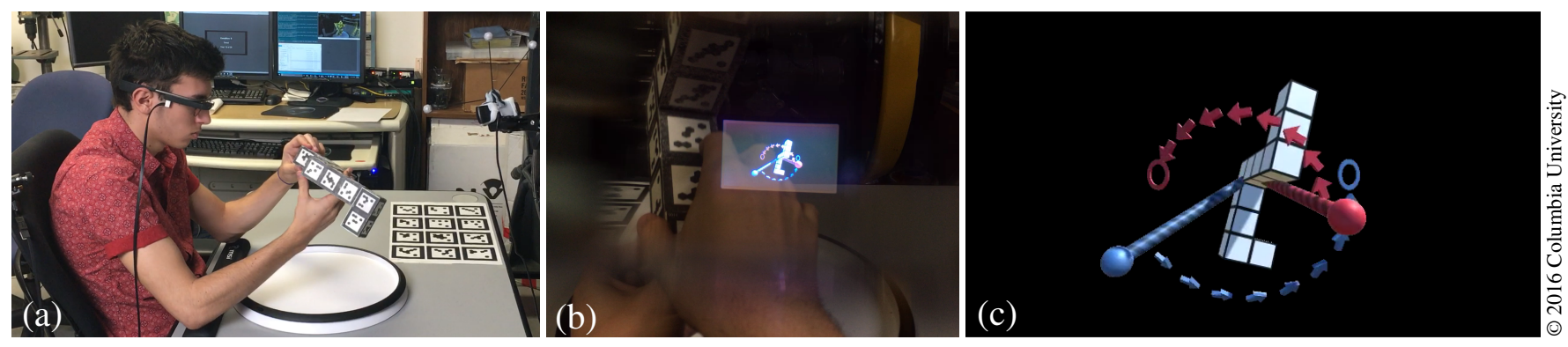

Figure 1: (a) Study participant manually orienting task object, guided by our system. (b) Participant's view of task and HANDLES visualization, photographed through Google Glass. (c) Screen capture of HANDLES visualization, rendered on Glass.

\begin{abstract}
Many tasks require that a user rotate an object to match a specific orientation in an external coordinate system. This includes tasks in which one object must be oriented relative to a second prior to assembly and tasks in which objects must be held in specific ways to inspect them. Research has investigated guidance mechanisms for some 6DOF tasks, using wide-field-of-view, stereoscopic virtual and augmented reality head-worn displays (HWDs). However, there has been relatively little work directed toward smaller fieldof-view lightweight monoscopic HWDs, such as Google Glass, which may remain more comfortable and less intrusive than stereoscopic HWDs in the near future. We have designed and implemented a novel visualization approach and three additional visualizations representing different paradigms for guiding unconstrained manual 3DOF rotation, targeting these monoscopic HWDs. We describe our exploration of these paradigms and present the results of a user study evaluating the relative performance of the visualizations and showing the advantages of our new approach.
\end{abstract}

\section{CCS Concepts}

-Computing methodologies $\rightarrow$ Mixed / augmented reality; Virtual reality; •Human-centered computing $\rightarrow$ Graphics input devices; Interaction techniques; Interaction design theory, concepts and paradigms; User interface design;

\section{Keywords}

Task assistance; Procedural task; 3D; UI; HWD.

Permission to make digital or hard copies of all or part of this work for personal or classroom use is granted without fee provided that copies are not made or distributed for profit or commercial advantage and that copies bear this notice and the full citation on the first page. Copyrights for components of this work owned by others than the author(s) must be honored. Abstracting with credit is permitted. To copy otherwise, or republish, to post on servers or to redistribute to lists, requires prior specific permission and/or a fee. Request permissions from permissions@acm.org.

SUI '16, October 15-16, 2016, Tokyo, Japan

(c) 2016 Copyright held by the owner/author(s). Publication rights licensed to ACM. ISBN 978-1-4503-4068-7/16/10 . .\$15.00

DOI: http://dx.doi.org/10.1145/2983310.2985764

\section{INTRODUCTION}

Many physical tasks require people to hold objects in specific orientations. In some cases, rotation tasks are simplified due to implicit physical constraints (e.g., a knob with discrete steps). However, numerous real-world situations, such as inspecting objects visually, or attaching one part to another, require unconstrained manual 3DOF rotation. Further, there are scenarios in which task objects or external references for alignment can be ambiguous; for example, a task object may be symmetric visually, but contain internal sensors, or a hand-held medical imaging device may need to be aligned with internal organs that are not seen directly. In these situations, providing guidance for rotating an object becomes a question of either conveying direction and magnitude explicitly, or annotating the environment to provide additional context for alignment.

Manuals, whether physical or virtual, often show different views of task objects and use annotations (e.g., connectors and arrows) to illustrate the required action [14]. However, these can be difficult to integrate, especially for complex, self-similar, or symmetric shapes, as mentioned above. Systems that present virtual instructions on a head-worn display (HWD) have been shown to help in transforming a rigid object to a predetermined position and orientation [12]. Such systems commonly employ basic virtual 3D UI elements such as arrows, animations, or clones of task objects as visual hints that guide users when performing manual operations (e.g., $[8,19,15,11,17,16,6])$. For example, based on this work, we can expect arrows to be suitable for showing a path of movement for a task object or body part. However, displaying paths as $3 \mathrm{D}$ arrows can be ambiguous for certain geometric projections, especially when viewed on monoscopic displays.

Even though these basic 3D UI elements have long been used in task guidance systems, we are not aware of any principled exploration of their effectiveness for real-time task assistance. In this paper, we begin to address this gap by presenting the design and comparative evaluation of a set of UI elements for a nontrivial rotation task, measuring their usability and effectiveness, and attempting to 
explain their relative effectiveness and trade-offs using cognitive science. In summary, we make four major contributions:

(1) We present HANDLES, a novel interaction and visualization approach to provide real-time guidance for unconstrained 3D rotation of hand-held objects (Figure 1). HANDLES was specifically developed to overcome shortcomings of existing approaches by providing persistent, clearly-visible alignment targets, and to work well on lightweight, monoscopic, small-FOV HWDs. (These devices, typified by Google Glass, Brother AiRScouter, and Vuzix M300, may remain more comfortable and less intrusive than widerFOV stereoscopic HWDs in the near future.) We show that users guided by HANDLES perform a nontrivial orientation task significantly faster compared to other techniques and tend to prefer it over the other techniques.

(2) We describe three additional orientation-guidance approaches that are built using variants of common UI elements found in existing orientation-guidance systems (e.g., 3D arrows and animation) and detail how we carefully fine-tuned these approaches to improve their usability.

(3) For each approach, we outline our exploration of the design space and justify our design choices and visualization parameters, based on usability feedback from extensive pilot studies. We highlight advantages and trade-offs of each approach and discuss how we expect it to perform, based on principles of human cognition.

(4) We report results and analysis from a formal user study that compares these four approaches with an unaided side-by-side representation of the static target orientation and a dynamic virtual proxy of the tracked object, addressing speed of performance (both overall speed, and the breakdown into initial ballistic rotation and subsequent fine-tuning), preference, and task load.

We believe that our detailed and principled exploration of the design space and our findings are valuable for the 3D HCI community, as our approaches can be used as a foundation for future eye-worn guidance systems.

\section{RELATED WORK}

Our visualizations are inspired by elements from a large body of work exploring task assistance using augmented reality (AR). Instructional manuals have long used arrows to depict rigid body transformations [14]. This approach has been adopted in computerbased documentation systems, including ones targeting AR. For example, arrows can cyclically move in a direction in which an object is to be translated [8] or interactively change in size and color to indicate direction and magnitude of a 1-DOF rotation needed to align two tracked workpieces [12]. Ghosting is another common visualization technique used in real-time AR task guidance systems to visualize workpiece placement (e.g., [11]). Ghosting and animation have also been used to provide visual hints on how to move (e.g., reel or shake) hand-held props to activate gestures in an AR system [27].

AR interfaces have been developed to guide users in matching gestures and poses with parts of their body. Freeman et al. [9] assist users in learning multi-touch gestures on a touchscreen by showing a partial shadow of the user's hands on screen. Sodhi et al. [22] guide a user in translating a single hand using a 3D arrow, a 3D path, or colored regions indicating movement direction projected directly on the user's hand. Anderson et al. [1] guide a user in moving their body by displaying augmentations over a mirror image of the user. Their visualization includes both a simple skeletal representation of the user's current and target poses, and a ribbon indicating the path the user should follow to achieve the target pose. These systems focus on hand and body manipulation directly, whereas our system focuses on guiding users in rotating a hand-held shape to a target orientation, allowing them to use their hands freely as they hold the tracked object.

We build on past work by focusing on a specific subtask-manual orientation of hand-held objects - aiming to improve upon existing techniques by providing users with continuous feedback designed to reduce cognitive load, facilitate corrective action, and provide confirmation once the target orientation is reached. Note that this is quite different from 3D applications on desktop systems that use separable rotation control widgets for one or more axes (e.g., [20]). Unlike those widgets, our techniques visualize the remaining rotation between a tracked object's current orientation and a target orientation. Further, our user is holding the tracked object with an unconstrained hand and cannot precisely manipulate the object to rotate only about a given axis as can be done with a desktop widget.

There is research on the standard mental rotation task, showing that people often spontaneously rotate a hand when solving the problem and that when they move their hand in the most efficient direction they perform better, but when forced to move their hand in the opposite direction they perform worse. That is, moving one's hands in a conceptually congruent way helps the user perform a mental transformation $[4,5,25,28]$. This research inspired the design of the nontrivial rotation task underlying our user study.

Oda et al. [17] used an annotation-based solution to guide a user to match a 6DOF pose specified by a remote subject matter expert (SME). The 6DOF pose of a manipulatable object was constrained by the physical properties of a fixture on which the user placed the object. Two types of orientation guidance were presented. In both, annotations on the manipulatable object and on the fixture provided a complete 6DOF specification for how the manipulatable object should rest on the fixture. In one technique, this was augmented with a replica of the virtual representation of the manipulatable object that animated in conjunction with the remote SME's control of the manipulatable object's replica in their virtual environment. In their work, rotation guidance was handled completely through matching annotations, with no additional guidance.

In an earlier poster [6], we briefly described a set of unevaluated techniques to help users manually rotate a tracked object to an arbitrary 3DOF orientation, tailored to lightweight, monocular HWDs. Building on this work, we ran extensive pilot tests, leading to a number of significant modifications to improve user performance, and resulting in a novel visualization described below. Finally, we conducted a formal evaluation to compare the new designs.

\section{IMPLEMENTATION}

We implemented our work in Unity 3D. While our visualizations are device-agnostic (i.e., can be rendered on various screen sizes and modalities-head-worn, hand-held, or desktop; monoscopic or stereoscopic), we developed and tested them on Google Glass. Because the current generation of lightweight, monoscopic HWDs typically have FOVs that are small and off-center, we created a virtual proxy of the tracked object, so that annotations can be rendered and registered relative to it instead of the real object (Figure $1 \mathrm{~b}$ ). The virtual proxy and visualizations are rendered from the perspective of a stationary virtual camera located near the head of the user, who is assumed to be sitting in place, as we ensured in our user study (Section 5.4.4). Arbitrary viewpoints could be accommodated with minor changes if head-tracking were available.

\subsection{Common Components}

A number of our visualizations incorporate curved 3D arrows to communicate $3 \mathrm{D}$ rotations, whose design evolved as we prototyped and tested our visualizations. Initially, we used simple 3D cylindrical arrows (Figure 2a), with a cone for the arrow head and a curved 


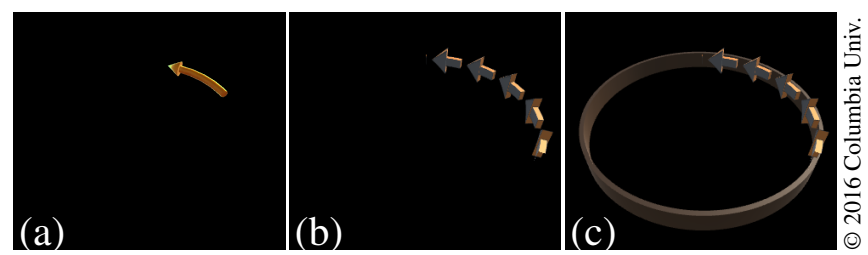

Figure 2: Arrow shape evolution. (a) A simple cylindrical, curved 3D arrow. (b) Repeating flattened 3D arrows increase amount of information encoded in arrow body. Walls facing towards the axis of rotation are colored differently to help disambiguate orientation. (c) Repeating flattened 3D arrows with semi-transparent ring to further clarify rotation axis.

cylinder for the body. During pilot studies, we noticed that the rotation axis implied by these arrows was often difficult to judge, especially when the magnitude of the arrow spanned less than $45^{\circ}$. To improve perceptibility, we switched to a flat, curved 3D arrow that had an extruded triangle for its head and an extruded rectangle for its body, which was essentially a curved version of commonly encountered 2D arrows, slightly thickened. This allowed us to provide more visual information about the implied rotation axis by increasing the width of the arrow. To further disambiguate the implied rotation axis, we applied a different color to the walls of the arrow that face towards the rotation axis, as opposed to one that face away from the rotation axis. The head of a flat 3D arrow can become indistinguishable from its body when the user is viewing the arrow from the side. Therefore, we made the arrow head into a pyramid with a single point at the tip and base that is wider than the cross-section of the body, to ensure that the head was distinguishable even when viewed from the side.

Even when the axis of rotation is clear, another issue we encountered was that in order to understand the direction of rotation, users had to constantly keep track of where the arrow head was. This problem was compounded when it was occluded by another object in the scene. To increase the amount of information encoded in the arrow body, we broke the single curved arrow into smaller ones along the same curve, analogous to a dashed line (Figure 2b).

Changing the length of the arrow body to represent the magnitude of remaining rotation created several issues. First, when the amount of the remaining rotation became small (i.e., the task was near completion), the amount of visual information available to the user was also lessened. This was counterproductive, since the user still needed as much information as possible to complete the finetuning stage. To address this problem, we added a ring (an extruded annulus) that contained the repeating arrows and did not disappear based on the magnitude of the remaining rotation. The ring is semitransparent and has the same hue as the arrow (Figure 2c). We note that there is a trade-off here between cluttering the scene, especially when multiple arrows are present, and not providing enough information; however, based on our testing, we believe the ring to be worth the visual space it occupies.

Another problem we faced with dynamically sized arrows was in their implementation, where we decided not to recalculate the positions of vertices and modify the vertex buffer in each frame. Instead, we left the full arrow geometry (i.e., $360^{\circ}$, the end of the body touching the tip of the head) untouched and implemented a custom pixel shader that took the remaining angle as a parameter and painted only those pixels that were within that angle of the tip.

\section{OUR APPROACHES}

In our implementation, the virtual proxy turns green whenever the target orientation is matched within a certain threshold. This is particularly important in the user study we conducted, to indicate to users that they have followed the instructions correctly and can move on to the next trial.

\subsection{SingleAxis Visualization}

SINGLEAXIS (Figure 3) applies the improved components of Section 3.1 to the OptimalAxis technique described by Elvezio et al. [6]. The core of the technique is inspired by Euler's rotation theorem [7], which dictates that any sequence of one or more rotations of a rigid body in 3D space is equivalent to an optimal rotation about a single axis. (Note that this axis is the unique single axis about which the differential rotation can be performed and therefore cannot, in general, be aligned with a major axis of the shape.)

In this visualization, a ring with small repeating dynamic rectangular 3D arrows is rendered around the virtual proxy, perpendicular to the axis of optimal rotation. In addition, a large cylinder, tied to the axis of optimal rotation, pierces the center of the virtual proxy. As the user rotates the tracked object, the axis and ring update to reflect the new axis and direction for a rotation from the tracked object's current orientation to the target orientation (relative to the world). As the magnitude of rotation gets smaller, the number of arrows decreases (where a single arrow will collapse from head to tail as it disappears), starting from the arrow furthest from the camera, and ending at the arrow closest to the camera (Figure 3).

During pilot studies, we observed that while the visualization worked quite well for large ballistic rotations, it became difficult for users to manage as the tracked object neared the target orientation (i.e., the fine-tuning stage). This was due to the rotational error between the current and target orientations changing drastically in direction, even from small adjustments made by the user. Visually, this resulted in the axis and ring swinging wildly around, making it difficult for users to understand how to execute the remaining rotation. To address this issue, we piloted a version of SINGLEAXIS that applied motion smoothing to the cylinder that represents the axis. Surprisingly, we found that smoothing negatively impacted user performance, especially during fine-tuning, where subtle changes to the rotation axis were not immediately represented. As users frequently deviate from the instructed axis during fine-tuning, the smoothed instructions would usually lag behind the user. Thus, we decided not to smooth the visualization during the user study described below.

In another design iteration, we displayed a static version of the original optimal axis of rotation. When the user deviated from this optimal axis, instead of showing the updated axis for the remaining rotation, we displayed a set of arrows that highlighted how to bring their current axis of rotation back to line up with the original. However, this solution was also disliked by pilot users, who now had to mentally resolve two separate rotations, instead of focusing on the single remaining rotation of the original version.

\subsection{Euler Visualization}

Another common way to describe an orientation in 3D space is Euler angles: a sequence of three elemental rotations (rotations about the three axes of an object's local coordinate system). Many objects are easily understood in terms of a particular coordinate system, whose axes can be chosen for the rotations. For the object shown in Figures 1 and 3-7, we use axes perpendicular to the faces of the cubes from which the object is composed. Because this visualization decomposes the rotation into three steps, each associ- 

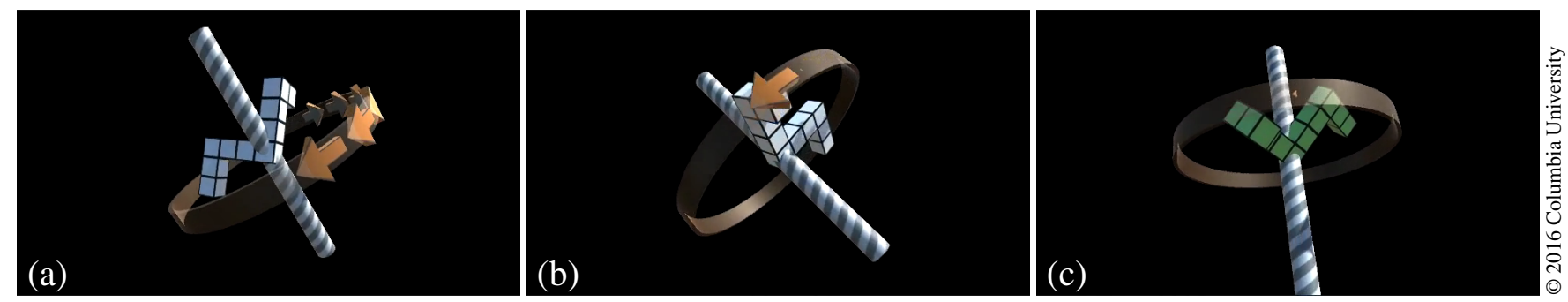

Figure 3: SingleAxis. (a) The remaining rotation is represented by a cylinder showing the axis of rotation and a set of dynamic arrows indicating the direction and magnitude of the remaining rotation. (b) As the user follows the visualization, the axis and arrows update to reflect the current optimal rotation from the current pose of the object to the target pose. (c) As the user nears the target pose, the arrows collapse into their arrowheads.
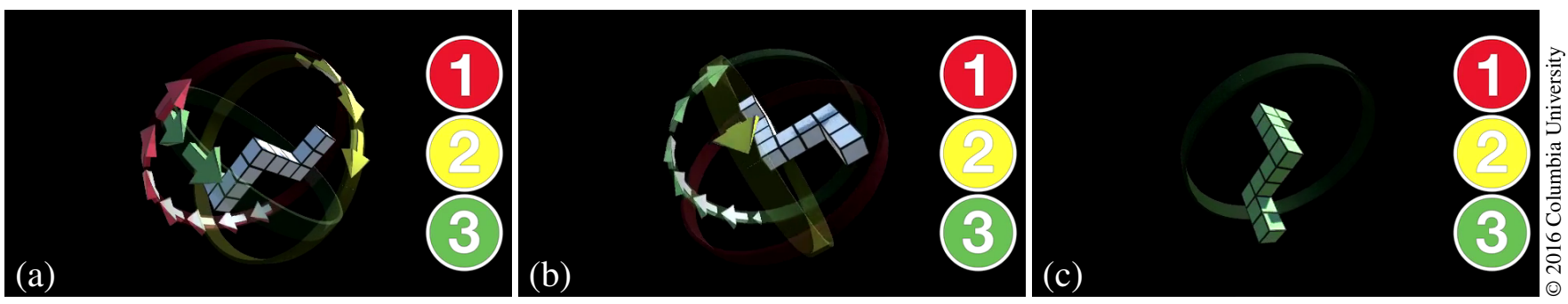

Figure 4: EULER. (a) The remaining rotation is represented by a set of three arrows showing the axes, direction, and magnitude of the remaining rotation. (b) As the user follows the visualization, in the order indicated by the colored numbered circles on the side, the arrows update to reflect the remaining rotation, per axis, from the current pose of the object to the target pose. (c) As the user nears the target pose, the arrows collapse into their arrowheads. If the user rotates away from the target about a particular axis, the arrows reappear.

ated with an easily recognizable axis, it might be easier to enact, especially by people with lower spatial ability (e.g., [24]).

Our EULER visualization (Figure 4) builds on the EulerAngles visualization [6]. In the previous EulerAngles, the axes of rotation were described by three cylindrical arrows, color-coded to represent the intended order of rotation based on the decomposition of the quaternion representing the remaining rotation. When pilot-testing this technique, we discovered that it was often difficult for participants to determine the direction of rotation about each axis, due to the fact that the user needed to search the cylinder that formed the shaft of the arrow for the arrow head. Additionally, it was possible that the virtual proxy itself would obscure the arrowhead, leading to situations where, with an untracked HWD such as Google Glass, it would be impossible to see the direction of the particular arrow without some initial trial and error.

To alleviate this, we used the improved components of Section 3.1 to introduce a number of new features. Instead of a single arrow per axis, we render a set of smaller arrows in a ring perpendicular to a particular principle axis. The smaller arrows disappear smoothly as described above. In addition, the front of the path is always anchored at the point on the ring closest to the virtual camera. The combination of these changes makes immediately clear, at all times, the intended direction of rotation per axis. Finally, upon nearing the completion threshold for a particular axis, the ring will disappear. It will return if the user breaks from the target orientation about a particular axis (Figure 4).

Since a sequence of 3D rotations is, in general, not commutative, there is a defined order for the axes about which the user should rotate the object when following the instructions. To remove the requirement that the user memorize the axis order, imposed in our previous work [6], we render three large icons on the screen showing the rotation order, represented by number and color.

\subsection{Animate Visualization}

Animation is a visualization technique frequently used to communicate motion or action. It became clear that animating the virtual proxy from its tracked (i.e., current) orientation to the target orientation was not ideal because the user had to wait until the animation finished and rewound to get feedback on current orientation. To provide feedback on both current orientation and desired motion simultaneously, our ANIMATE visualization (Figure 5) adds a second, animating copy of the virtual proxy to the scene. We quickly realized that the placement of this animating copy had a significant impact on user performance. Initially, since our task is rotationonly, the animating copy overlapped with the virtual proxy and made it difficult to distinguish one from the other. In our following iterations, we tried rendering the animating copy and the virtual proxy side-by-side, similar to our earlier work [6]. This proved to be suboptimal, especially in the fine-tuning stage, because it required users to detect differences between two objects that have similar orientations, but are spatially set apart. Going back to a co-located design, we addressed the occlusion and disambiguation issues caused by overlapping, by modifying the transparency of the animating replica to 50\% and changing its outline from solid black lines to dashed grey lines (Figure 5). This faded visual is known as ghosting, an illustrative technique used in comics [13] where an object is rendered as semitransparent to represent its past or future state, and in previous visualizations (e.g., [27, 11]).

Another subtle, yet important, design decision was the timing and speed of the animation. We wanted to provide users with continuous feedback, so it was a natural decision to repeat the anima- 

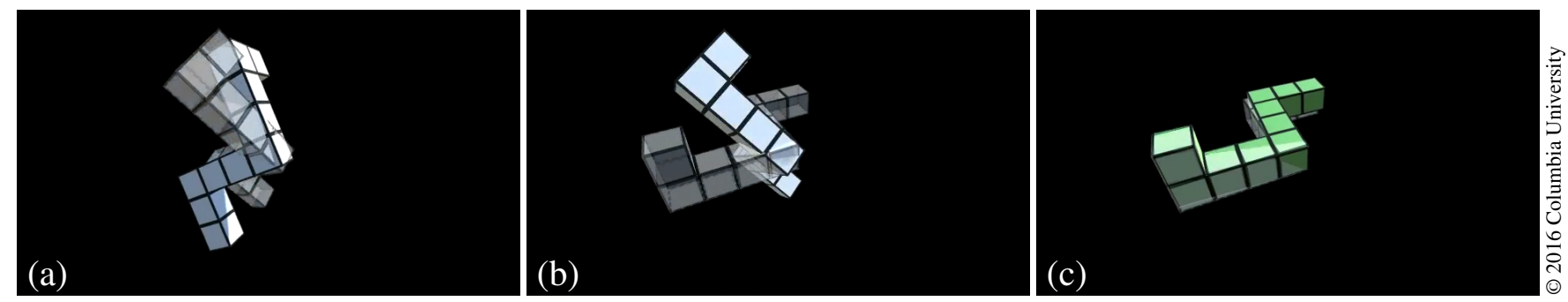

Figure 5: Animate. (a) The remaining rotation is represented by an animating clone of the virtual proxy, which rotates from the current orientation of the tracked object, to the destination orientation. (b) As the user follows the visualization, the looping animation will begin from the latest orientation of the tracked object. (c) As the user nears the target pose, the frequency and speed of the animated object will increase, until the task is complete.
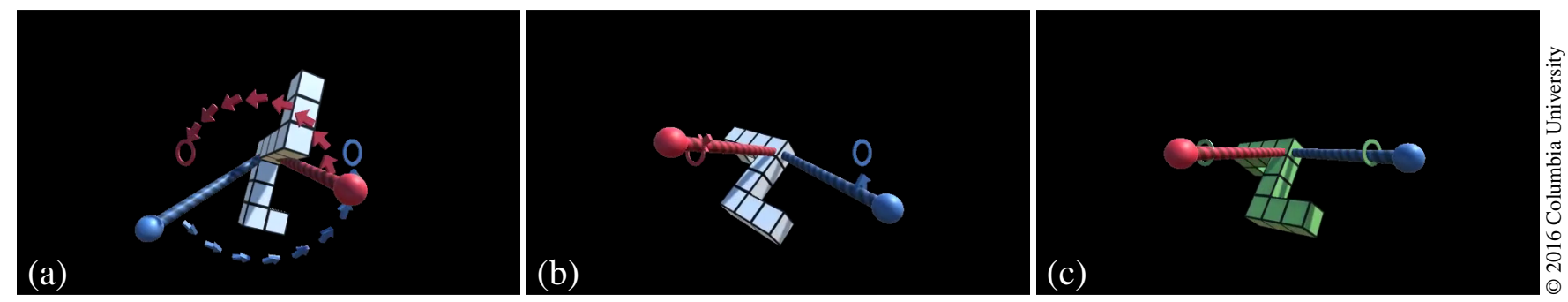

Figure 6: HANDLES. (a) The target orientation is directly represented by a set of two colored tori. Two colored poles extend from the center of the virtual proxy, and the user must try to align each pole with its matching torus. A set of arrows show the rotational path from each pole to its corresponding torus. (b) As the user nears the target pose, the arrows update to show the current rotational path from each pole to its corresponding torus. (c) Both handles have been aligned, the tori turn green, and the task is complete.

tion once the animating copy arrived at the target orientation by rewinding the animating copy to the tracked object's current orientation. We implemented an ease-in, ease-out interpolator to make the beginning and end of the animation less visually jarring and abrupt for the user. Setting the duration of each animation cycle to a constant value did not make much sense, since that would require the animating copy to move more slowly as the tracked object neared its target, which pilot users found frustrating.

Setting the speed of the animation seemed like a better idea and we found a rotational speed of $90^{\circ}$ per second to be comfortable based on pilot tests. This ensured that when the tracked object was near its target orientation, the animation took less time and therefore repeated more frequently. However, when the frequency got too high, it became less helpful because it was hard to distinguish between the animation progressing forward and rewinding back to the current orientation. To address that issue, we introduced a 0.5 second gap between animations. Finally, we clamped the total animation duration to be between 0.2 and 2 seconds.

\subsection{Handles Visualization}

The HANDLES visualization (Figure 6) builds on the key insight that orientation in 3D space is commonly parametrized by two different directions (e.g., virtual cameras in computer graphics are often defined by specifying a non-collinear pair of vectors: lookat and up). In HANDLES, these non-collinear directions are represented by poles extending from the center of the virtual proxy, which look like physical handles. Each pole's target orientation is represented as a torus whose hole is just wide enough for its pole to go through. These tori are persistently placed in direct view of the user to avoid occlusion. Additionally, each pole is connected to its corresponding torus by a set of color-coded arrows to indicate the direction of rotation necessary to achieve the target orientation (Figure 6).

The 2-Point visualization, developed in our earlier work [6], required the user to align a pair of points attached to the virtual proxy, represented by cones, with a pair of corresponding points that are fixed in space, represented by target spheres. In piloting an implementation of 2-Point, we found that it had shortcomings that severely limited its effectiveness. Users often tried to translate the shape so that a cone/sphere pair would align, even after being instructed that translation was not being tracked. The locations of the target spheres depended on the specific task in a seemingly arbitrary way. Additionally, users complained that the cones and spheres were too small, making it difficult to distinguish which way the cones were pointing, and the enveloping sphere made it difficult to see the cones and the virtual representation of the main object contained within.

Since we wanted to make the poles look like physical handles that are rigidly attached to the virtual proxy, we added a spherical knob to the end of each pole to (a) bolster the metaphor that the poles are handles that can be grabbed and moved, and (b) provide occlusion and perspective depth cues, which could be especially beneficial when the handles are near their targets (i.e., during the fine-tuning stage, which was an issue mentioned during pilot tests).

An important design question was where to attach handles to the virtual proxy. Initially, we attached them along the major axes of the shape, but for certain target orientations, this caused the tori to face away from the user, possibly occluded by the virtual proxy. Since the visibility of the tori is crucial for this task, especially in the fine-tuning stage, we wanted to guarantee that they are always front and center and clearly visible to the user.

To that end, we developed a heuristic in which we start with a vector connecting the centroid of the virtual proxy to the center of 
projection of the virtual camera, rotate it $30^{\circ}$ about the virtual camera's up-vector (clockwise for the first torus, counterclockwise for the second), and pick the intersection of that rotated vector with a spherical hull that contained the virtual proxy (to ensure that the virtual proxy would never touch or occlude the tori in any orientation). Since we had a stationary virtual camera pointed directly at the virtual proxy, this heuristic gave us two locations that are projected to lie on the horizontal centerline of the screen. Picking where the tori end up first meant that the poles would have to be attached in different orientations relative to the virtual proxy for each new target pose, which was calculated during initialization by applying the inverse of the rotation between the current orientation to the target orientation, to the tori positions.

To provide users with a sense of which direction to move the object, we added arrows that connected each handle to its corresponding torus. Initially, we used the same curved arrows that we used in other visualizations, which depicted the shortest path along the sphere from handle location to torus location. During pilot tests, we noticed users getting frustrated when following the shortest rotation between one of the handles and its torus worsened the alignment between the other handle and its torus. To alleviate this frustration, we replaced the arrows that traced the shortest path for each individual handle with "cookie-crumb" arrows that trace the ideal path of the handles when both of them are moved towards their target simultaneously (i.e., by following the single-axis optimal rotation from the current orientation to the target orientation). Similar to our other visualizations, the trail of arrows gets shorter as the user rotates the object and the remaining angle gets smaller.

To provide visual feedback for when the alignment is complete, we relied on color. Specifically, when a handle enters its corresponding torus, that torus turns green to indicate proper alignment for that pair. Once one of the handle-torus pairs is aligned, the user needs to bring the second handle into its corresponding torus while holding the first handle in place, which can be achieved by executing a 1 DOF rotation (Figure $6 b-c$ ).

\section{USER STUDY}

We conducted a formal user study to compare the performance of our new techniques, in addition to a control condition described below. For our task object, we created an abstract object similar to those used by Shepard and Metzler in research on mental rotation [21]. Our object consists of ten 1.75-inch wooden cubes attached face-to-face to form a rigid structure with three right-angled "elbows" (Figure 1a). This type of object is especially suited for rotation tasks because (a) it cannot be transformed into itself by any reflection or rotation (short of $360^{\circ}$ ) and (b) cognitive science research has shown that it is difficult to mentally rotate [21, 23].

We required that the accuracy with which the participant performed each trial be as close to the correct pose as possible for the trial to end; therefore, we compared only time, not accuracy. We settled on a threshold of $8^{\circ}$ by incrementally loosening a tighter threshold until pilot users were able to satisfy it consistently. Tighter constraints were especially difficult for visualizations that provide little or no feedback during fine-tuning (e.g., STATIC).

\subsection{Control Condition}

To determine the effectiveness of the techniques described above, with respect to a simple baseline, we developed a fifth technique, STATIC, which showed only the static target orientation next to an updating representation of the virtual proxy's current orientation (Figure 7). The virtual proxy would update as the user rotated the shape. When the target orientation was achieved, the virtual proxy

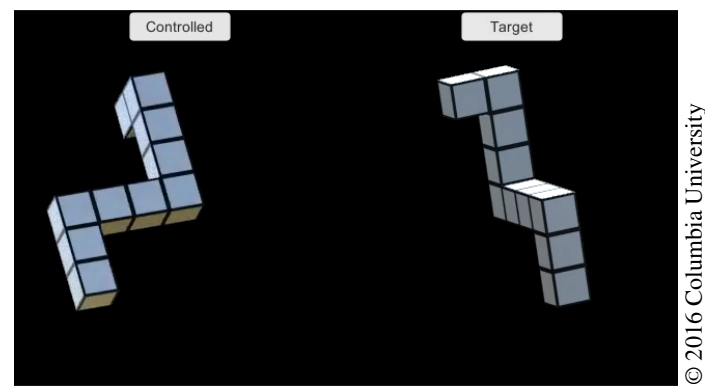

Figure 7: STatic. Control Condition.

would turn green. This provided a simple control condition to use in the user study described below.

\subsection{Pilot Studies}

Pilot studies were instrumental in guiding the evolution of the techniques, as described above, and helped us refine study parameters. In particular, HANDLES was designed to overcome specific shortcomings of existing techniques by providing a persistent target and ensuring high visibility of landmarks and targets.

\subsection{Hypotheses}

Based on an analysis of the tasks and extensive design iterations informed by and tested in pilot studies, we formulated the following five hypotheses:

\section{H1. HANDLES will be the fastest technique. \\ $\mathrm{H} 2$. HANDLES will be the preferred technique. \\ H3. SINGLEAXIS and STATIC will be less preferred compared to HANDles, Animate, and Euler. \\ H4. HANDLES will be fastest for fine-tuning. \\ H5. EULER will be preferred by users with low spatial ability.}

\subsubsection{Rationale}

$\mathrm{H1}$ : In presenting the rotation instruction, HANDLES is the only visualization that presents both a persistent view of the target orientation (relative to the world coordinate system) and a view of the optimal transformation needed to get to the target orientation from the tracked object's current pose. ANIMATE shows the latter transformation, but to avoid cluttering the virtual scene with a third model of the virtual proxy, does not always show the target pose (besides the pause at the end of the animation loop). As a result, it is possible that the user will need to wait for a certain amount of time to comprehend the rotation instruction. SINGLEAXIS also shows the optimal path, but during pilot testing we found that due to limitations inherent to the small monoscopic display, it could be difficult to disambiguate certain rotation instructions, leading to situations where users would lose time trying to understand the direction of the instruction. EULER is similar to SINGLEAXIS, but breaks the transformation into three steps, further lengthening total trial time. Last, during pilot testing, we found that showing the target orientation at all times (and ensuring that the torii are placed at consistent locations relative to the user's line of sight), allowed the user to associate the destination target with a consistent position in the real world. In consideration of all four points above, we hypothesize that HANDLES will be the fastest condition in total completion time.

H2: Since the core components in HANDLES, the poles and tori, allow a user to easily determine the target orientation, and the connecting arrows show the remaining rotation, the user should be able 
to determine their next action by a short glance. A user may need to watch a few cycles of ANIMATE to completely understand the rotation instruction, potentially waiting for the animation to loop back to the beginning to follow along. SINGLEAXIS should work well with ballistic movements, but due to potentially radical motions of the axis in the fine-tuning stage, users may become frustrated as they try to complete a small rotation. EULER works consistently throughout a rotation task, but the required axis completion order makes acceleration difficult; thus, more skilled users could potentially be limited in performance. Consequently, we believe HANDLES would be the preferred condition.

H3: As the tracked object nears the target orientation, the axiscylinder is highly sensitive to small movements that change the rotation axis. If the user is only a few degrees from the angular completion threshold, but drifts slightly in following the rotation instruction, it is possible that they stop progressing towards the goal as they try to adjust to the new rotation axis. As a result, users could potentially become confused and frustrated. Secondly, since STATIC provides no instruction (other than a visualization of the target orientation), users may struggle trying to match the pose in the given threshold, when fine-tuning. As the target object is positionally offset from the virtual proxy, and rendered on a small display, it may be difficult to discern the exact orientation difference between the tracked and target objects. As a result, we expect users will rate either SINGLEAXIS or STATIC as least preferred.

$\mathrm{H} 4$ : In the fine-tuning stage, the remaining rotation is within $16^{\circ}$ of the tracked object's current orientation, which for many of the visualizations may result in a very small or slight change. As explained in the rationale for $\mathrm{H} 1, \mathrm{H} 2$, and $\mathrm{H} 3$, HANDLES always shows both the target orientation and the remaining rotation. This gives users two forms of feedback to use in the fine-tuning stage. If one is not clear, the other may still provide enough information to discern the correct instruction. ANIMATE shows the exact motion needed to complete the task, but as the animated object is overlaid on the virtual proxy, and rendered on a small display, it is possible that a user simply may not be able to see the animated object clearly enough to discern the proper action. SINGLEAXIS has the issue of the fast-moving rotation axis cylinder, as described in H3. This makes completing the fine-tuning task potentially difficult. EULER shows the remaining rotation per axis clearly, but since breaking the completion status of a particular axis may require that a user recomplete it before continuing, it is possible that users spend a nontrivial amount of time in fine-tuning dealing with previously completed axes.

H5: Each of the visualizations require that a user be able to mentally map the instruction to a motor action in rotating the tracked object. For SINGLEAXIS, ANIMATE, and HANDLES, the instructed action may be a rotation about an axis that does not line up with a natural axis of the held object, and that may require an unintuitive motion. EULER instructs the user by presenting the rotation guidance through a set of three transformations about axes fixed to the virtual proxy. This allows the user to focus on one axis per motion, potentially rotating the shape to a more easily manipulated orientation before beginning. As EULER does not require the user to map the rotation axis to one not attached to the tracked object, we expect that users with low spatial ability who may struggle with this particular mapping to prefer EULER.

\subsection{Methods}

\subsubsection{Participants}

We recruited 17 participants from our institution (9 female), 1932 years old (average 23), through email and posted flyers. Partic- ipants attended a single-session experiment. Five participants had previous experience with AR, and none had any familiarity with our techniques.

\subsubsection{Equipment}

Participants wore a Google Glass Explorer Edition running Unity Remote 4. This Android app allows Glass to display visual output provided through USB 2.0 from our software running in Unity 5.3.3 on a computer powered by an Intel i7-3770k with $16 \mathrm{~GB}$ of RAM and an Nvidia GeForce GTX 780. (Our software can also run in Unity directly on Glass, but causes it to overheat too quickly to complete the study.) The object held by the user was tracked using a Logitech c920 camera (visible at the right of Figure 1a), using tracking software in the Canon MREAL Platform, running on the same computer. The Logitech camera tracked both the held object and a fiducial array on the table where the user was seated, in order to ground the environment. The software running on Google Glass communicated to the MREAL Platform tracking application through a Unity application server, which ran on the same computer as the MREAL Platform software. Additionally, a foot-pedal was placed under the participant's table, and used to progress through the study.

\subsubsection{Design}

Since it was possible that certain rotations would be easier to maneuver than others, depending on how the user was holding the tracked object, the user study was designed to select from one of four possible rotations $\left(80^{\circ}, 100^{\circ}, 120^{\circ}, 140^{\circ}\right)$ that would build on the target orientation in the preceding trial. There were an equal number of each of the possible rotation magnitudes across a single condition. Each trial would also generate a random rotation axis.

Trials were blocked by technique and randomized by rotation axis. Each block included four practice trials and 16 timed trials. The presentation order of the techniques was counterbalanced across participants to minimize bias due to learning.

\subsubsection{Procedure}

Participants were welcomed by the study coordinators and given the PseudoIsochromatic Plate (PIP) Color vision test to screen for color blindness, the Stereo Optical Co. Inc. Stereo Fly Test (SFT) to screen for stereo vision, and the Vandenberg-Kuse Mental Rotation Test (MRT) [23] to screen for spatial ability. All participants passed the PIP test. 12 participants passed the SFT, four had weak stereo vision, and one failed the test.

After completing the tests, the participant was seated in a chair pushed up to a table and instructed to rest their elbows on a gel wrist pad (1a) while holding the task object with both hands. These constraints ensured that their view of the virtual proxy was consistent with their view of the physical object. The participant was then introduced to the study and given an explanation of each of the techniques, with a small hands-on demonstration session for each technique (consisting of two practice rotations using the technique). Before the first condition, the participant was given a detailed explanation of each interaction technique.

At the start of each trial, the participant was shown the virtual proxy of the tracked object and the visual components of the current technique. The participant was instructed to match the 3DOF pose demonstrated using the current technique. The participant was instructed to press a button on a foot-pedal controller when the virtual proxy turned green, indicating that the target orientation had been met. The system prevented the user from completing the trial by pressing the button if the tracked object had not yet entered the acceptable range for the trial (as explained in Section 5). Once the 
trial was complete, the participant was instructed to hold their pose for 1.5 seconds as they entered the next trial. Throughout the study, the positions and orientations the tracked object were recorded.

Participants were asked to complete a three-part questionnaire before, during, and after the study, assessing the five techniques. The questionnaire included an unweighted NASA TLX, a request to rank the techniques from 1 ("Most Preferred") to 5 ("Least Preferred"), and room for free-form comments.

\section{RESULTS}

Each participant completed a total of 80 timed trials ( 5 conditions $\times 16$ timed trials). We evaluated our hypotheses for significance using a Bonferroni-corrected $\alpha$ of $0.01(0.05 / 5)$.

\subsection{Task Duration}

\subsubsection{Outliers}

We identified outliers in terms of task duration using Tukey's outlier filter. We chose a standard threshold (1.5 times IQR per user per condition), resulting in 5.0\% (68 of 1360 trials: 13 ANIMATE, 13 EULER, 16 SINGLEAXIS, 15 STATIC, and 11 HANDLES) of our collected data being excluded from the rest of our analysis. Outliers resulted from occasional unstable tracking, connectivity issues, overheated HWD, or external issues (ringing cellphone, loose contact lens); in a few cases, users could not figure out the right answer and opted out, especially for STATIC.

\subsubsection{Analysis}

Our task completion metric is similar to reaction time (RT) data commonly analyzed in psychology experiments, in that we measure the time it takes users to react in response to a visual stimulus. Traditional analysis of variance (ANOVA) methods are generally not well-suited to RT data [26], because RT distributions are typically not Gaussian: they often have a long tail on the right, presumably due to confounding factors such as fatigue and external distractions. Before we began our analysis, we quickly confirmed that our task-completion data exhibited similar non-normality by fitting a linear model and visually inspecting the residual plots, which in fact showed obvious deviations from normality.

One widely adopted method for analyzing such heavily skewed RT data is to transform it into a reaction rate (analogous to speed) by taking the reciprocal $(1 / x)$ and then fit it with a linear-mixedeffects (LME) model to identify significant effects by adding and removing factors [2]. Using $\mathrm{R}$ [18] and its lme4 package [3], we fit an LME model to our task-duration variable as a function of visualization condition (fixed effect) and participant (random effect). Compared to a base model with a random slope and participant as a random effect, a Kenward-Roger corrected $F$-test showed that visualization condition was significant as a fixed effect $\left(F_{(1,271)}=\right.$ $119.16, p<.001$ ) (Figure 8a). A pairwise least-squares means comparison revealed that our participants were fastest using HANDLES, followed by ANimATE, SingleAxis, STATIC, and EulER, in that order, where all pairwise differences were statistically significant (Table 1). These findings validated H1.

\subsubsection{Ballistic Approach vs. Fine-Tuning}

In H4, we hypothesized that HANDLES would lead to faster task completion times compared to other techniques. Subdividing performance into a ballistic phase, followed by a visual feedback phase for "fine tuning" [10], We believed HANDLES would be faster because of its emphasis on providing visible, persistent feedback to help facilitate fine-tuning. In contrast to other techniques that rely on displaying the difference between current and target orientation,

\begin{tabular}{llr}
\hline Pairwise Comparison & t-statistic & p-value \\
\hline HANDLES vs. ANIMATE & $t_{(1,271)}=3.67$ & $p<.001$ \\
ANIMATE vs. SINGLEAXIS & $t_{(1,271)}=4.98$ & $p<.001$ \\
SINGLEAXIS vs. STATIC & $t_{(1,271)}=4.49$ & $p<.001$ \\
STATIC vs. EULER & $t_{(1,271)}=6.34$ & $p<.001$ \\
\hline
\end{tabular}

Table 1: Task duration-Pairwise comparisons

in HANDLES the difference between current orientation and target orientation is small during fine-tuning.

To confirm this part of our hypothesis, we separated the overall completion time into two subtasks, as shown in Figure 8(a): ballistic approach and fine-tuning, following a simple distance thresholding heuristic. We counted the amount of time for each trial that was spent where the user-tracked object was more than a certain threshold away from the target orientation as ballistic approach and the rest of the time (i.e., when the tracked object's orientation was within that threshold) as fine-tuning. We chose $16^{\circ}$ as our threshold for this analysis, which we arrived at by doubling our completion acceptance threshold of $8^{\circ}$.

Similar to how we analyzed overall task duration, we fitted two separate LME models to model reciprocals of ballistic and finetuning durations (i.e., ballistic and fine-tuning rates) as a function of visualization condition (fixed effect) and participant (random effect).

Ballistic Approach: For the ballistic approach, compared to a base model with a random slope and participant as a random effect, a Kenward-Roger corrected $F$-test showed that visualization condition was significant as a fixed effect $\left(F_{(1,271)}=111.73, p<\right.$ $.001)$. A pairwise least-squares means comparison revealed that there were significant differences between all pairs except HANDLES-ANIMATE at $p<.01$. In other words, HANDLES and ANIMATE were fastest, followed by SINGLEAXIS, STATIC, and EULER, in that order.

Fine-Tuning: For fine-tuning, compared to a base model with a random slope and participant as a random effect, a Kenward-Roger corrected $F$-test showed that visualization condition was significant as a fixed effect $\left(F_{(1,271)}=31.13, p<.001\right)$. A pairwise leastsquares means comparison revealed that there were significant differences between all pairs except ANIMATE-SINGLEAXIS, ANIMATE-EULER, and STATIC-EULER at $p<.01$. In other words, HANDLES was fastest for fine-tuning, followed by ANIMATE and SingleAxis, followed by STATIC and EULER, which confirms H4.

\subsection{User Feedback}

\subsubsection{Technique Rankings}

A majority of participants $(9,53 \%)$ ranked HANDLES as their most preferred condition (Figure 8b), supporting H2. Three participants (18\%) chose ANIMATE and EULER each, two participants (12\%) chose STATIC, and none chose SINGLEAXIS as their favorite. On the opposite end of the spectrum, SINGLEAXIS was chosen as the least favorite 8 times (53\%), followed by EULER with four times (24\%). On average, HANDLES was ranked highest, followed by Animate, SingleAxis, Static, and EulER. A Friedman test confirmed that our participants' differential preference between techniques was statistically significant, $\chi_{(4)}^{2}=17.459, p<$ .01 .

In H2, we hypothesized that HANDLES would be the most preferred technique. A post-hoc pairwise comparison using Nemenyi's procedure showed that the only statistically significant difference 


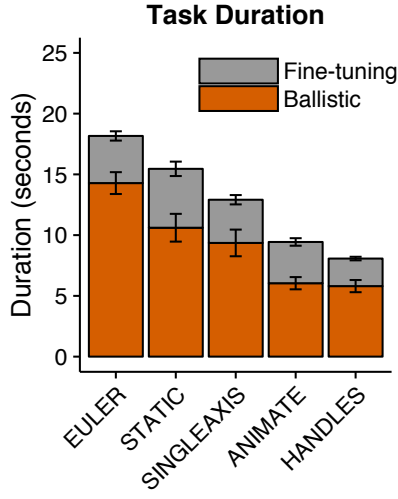

(a)

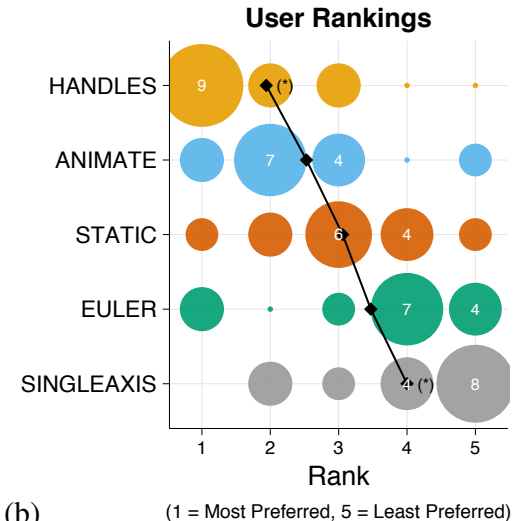

(b)

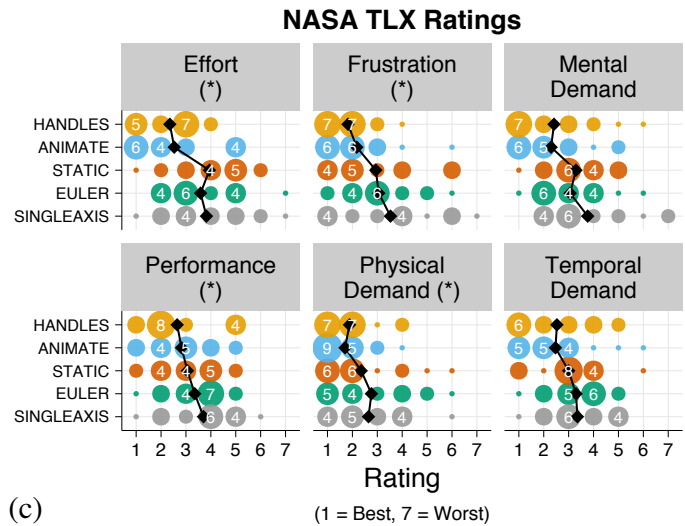

(c)

Figure 8: User Study: (a) Task duration per technique. (b) User rankings and (c) NASA TLX ratings per technique. Black diamonds shows average ranking/rating per technique. (*) denotes significance at $p<.01$.

in rankings was between HandLES vs. SingleAxis, $p<.01$. The differences between HANDLES vs. EULER and ANIMATE vs. SINGLEAXIS were nearly significant $(p=.039$ and $p=.052$, respectively), but above our Bonferroni-adjusted $\alpha$ of .01, supporting but not confirming $\mathrm{H} 2$.

Qualitative user feedback highlighted instances where participants found HANDLES to be generally more preferred for finetuning (e.g., "HANDLES was very accurate and didn't have changing parameters," "HANDLES was the best for putting the object in the exact position that the program wanted," "HANDLES is the best because it really helps with the small movements").

On the opposite end of the spectrum, SINGLEAXIS was generally rated as least preferred and participants reported frustration during fine-tuning (e.g., "SINGLEAXIS was frustrating, since the bar seemed to move very erratically with small movements, so it took a lot of concentration to do the fine movements near the target"). While the first part of H3 (i.e., SINGLEAXIS would be less preferred compared to HANDLES, ANIMATE, and EULER) was supported in our data, it was not confirmed because not all pairwise differences had $p<.01$.

In H3, we also hypothesized that STATIC would be less preferred compared to Handles, Animate, and Euler. Surprisingly, STATIC was generally ranked higher than EULER in terms of overall preference, which meant that the second part of $\mathrm{H} 3$ should be rejected. Four participants $(24 \%)$ ranked EULER as their least preferred technique and another six (35\%) participants ranked it as their second least preferred.

In H5, we hypothesized that EULER would be preferred by participants with low spatial ability. The three users who ranked it as their most preferred technique had either above or close to average MRT scores of 8, 12, 14 (out of 20), failing to support H5.

To understand why many users found EULER challenging, we examined the questionnaire comments from participants who did not prefer EulER. Common themes were that the need to perform three sequential rotations along the major axes of the tracked object was onerous and having to track three arrows at once was challenging (e.g., "in aligning one axis, the other pre-aligned axes may drift and cause some confusion," "Too many rings, and too many changing rotations," "Holding the rotation in one axis constant while rotating the others was challenging. Also, following the order of rotation was not instinctual.”).

\subsubsection{NASA TLX}

When we analyzed the results from the unweighted NASA TLX questionnaire (Figure 8c), a Friedman test confirmed that technique was a significant factor for Mental Demand, Physical Demand, Effort, and Frustration at $p<.01$. (The $p$ values for Temporal Demand and Performance were .081 and .051, respectively.)

Post-hoc pairwise comparison using Nemenyi's procedure indicated that SINGLEAXIS was rated as being significantly more mentally demanding compared to both HANDLES and ANIMATE $(p<.01)$. HANDLES was perceived to require less effort than both STATIC and SingleAxis, but the $p$ values for the pairwise comparisons were just above our Bonferroni-adjusted $\alpha<.01$ ( $p=.017$ in both cases). Similarly, HANDLES was rated as less frustrating compared to SINGLEAXIS and EULER, but that difference was also not significant $(p=.028$ and $p=.045$, respectively). There were no significant pairwise differences for Physical Demand.

\section{CONCLUSIONS AND FUTURE WORK}

We have described one new visualization (HANDLES) and three visualizations that improve upon existing approaches for guiding a user in rotating an object to match a specified 3DOF orientation. In addition, we have presented the results of a user study comparing the effectiveness of these visualizations, when viewed on Google Glass, a small field-of-view, monoscopic, off-center HWD. Our study found that HANDLES was significantly faster than and trended toward being preferred over the other techniques.

ANIMATE and STATIC include a representation of the object in the desired orientation, encouraging comparison of the current orientation of the object with the desired orientation. In contrast, SINGLEAXIS, EULER, and HANDLES provide virtual annotations as guidance (e.g., arrows, handles, and tori), requiring the user to attend only to those and shifting the task from spatial transformation to perceptual tracking. Despite this shift away from spatial thinking, HANDLES still provides a spatial representation of the final pose via its tori, which might explain why users were able to perform both ballistic and fine-tuning movements quickly. This was highlighted by several participants in their qualitative feedback (e.g., "Having the poles as a guide really helps. Don't have to think, can just get by with spatial intuition," "I just thought about how to put the sticks to the ring," "The two guides were very useful in determining the target position," "With HANDLES, I did not have to observe the orientation of the object I was holding to solve the trial").

While the visualizations were designed with small-FOV mono- 
scopic HWDs in mind, they should also work well with wider-FOV stereoscopic HWDs. On a stereoscopic AR display, the virtual proxy could be eliminated and our visualizations could be registered with and rendered directly on the user's view of the task object. However, it is possible that relative performance among the visualizations may change with increased FOV and stereoscopy; for example, EULER might perform better relative to some of the other techniques, when not confined to a small monoscopic display. Further, using tracked, registered AR on a wider-FOV display might also result in different relative performance across the techniques. Thus, we believe it will be useful to run new studies to assess the relative performance of the techniques when used with different display technologies.

We would also like to build in color profiles that could be applied to accommodate users with color-vision deficiencies. (We note that while our current version of EULER would be problematic for users with red-green colorblindness, all of our study participants passed the PIP test.)

While this work focused on unconstrained 3DOF rotation, it is possible that a number of the visualizations may work when completing 6DOF transformation tasks. Similarly, looking at specific types of constrained rotations (e.g., camera-plane vs. horizontal or vertical planes; or roll vs. pitch or yaw) could reveal interesting differences between techniques. Thus, we would like to explore how our visualizations may be modified or combined with other visualizations to support translation and constrained rotations, allowing them to address a full range of rigid-body transformations.

\section{ACKNOWLEDGMENTS}

This research was funded in part by NSF Grants IIS-1514429 and IIS-1513841. We thank Google for the gift of Glass, Canon U.S.A. Inc. for loaning the MR Platform software suite, and Minhaz Palasara and Yujin Ariza for their contributions to our system.

\section{REFERENCES}

[1] F. Anderson, T. Grossman, J. Matejka, and G. Fitzmaurice. YouMove: Enhancing Movement Training with an Augmented Reality Mirror. In Proc. ACM UIST, pages 311-320, New York, NY, USA, 2013.

[2] R. H. Baayen and P. Milin. Analyzing reaction times. Int. Jnl. Psych. Res., 3(2):12-28, 2015.

[3] D. Bates, M. Mächler, B. Bolker, and S. Walker. Fitting Linear Mixed-Effects Models Using lme4. Journal of Statistical Software, 67(1):1-48, 2015.

[4] M. Chu and S. Kita. Spontaneous gestures during mental rotation tasks: insights into the microdevelopment of the motor strategy. Jnl Expr. Psych.: Gen., 137(4):706, 2008.

[5] M. Chu and S. Kita. The nature of gestures' beneficial role in spatial problem solving. Journal of Experimental Psychology: General, 140(1):102, 2011.

[6] C. Elvezio, M. Sukan, S. Feiner, and B. Tversky. [POSTER] Interactive Visualizations for Monoscopic Eyewear to Assist in Manually Orienting Objects in 3D. In Proc. IEEE ISMAR, pages 180-181, 2015.

[7] L. Euler. Formulae generales pro translatione quacunque corporum rigidorum. Novi Acad. Sci. Petrop, 20:189-207, 1775.

[8] S. Feiner, B. Macintyre, and D. Seligmann. Knowledge-based augmented reality. Commun. ACM, 36(7):53-62, 1993.

[9] D. Freeman, H. Benko, M. R. Morris, and D. Wigdor. ShadowGuides: Visualizations for In-situ Learning of
Multi-touch and Whole-hand Gestures. In Proc. ACM ITS, pages 165-172, New York, NY, USA, 2009.

[10] K.-C. Gan and E. R. Hoffmann. Geometrical conditions for ballistic and visually controlled movements. Ergonomics, 31(5):829-839, 1988.

[11] A. Gupta, D. Fox, B. Curless, and M. Cohen. DuploTrack: A Real-time System for Authoring and Guiding Duplo Block Assembly. In Proc. ACM UIST, pages 389-402, New York, NY, USA, 2012.

[12] S. J. Henderson and S. K. Feiner. Augmented reality in the psychomotor phase of a procedural task. In Proc. IEEE ISMAR, pages 191-200, Los Alamitos, CA, USA, 2011.

[13] S. McCloud. Understanding Comics: The Invisible Art. William Morrow Paperbacks, New York, reprint edition edition, 1994.

[14] P. Mijksenaar and P. Westendorp. Open here: the art of instructional design. Joost Elffers Books, 1999.

[15] A. Miller, B. White, E. Charbonneau, Z. Kanzler, and J. J. LaViola Jr. Interactive 3D Model Acquisition and Tracking of Building Block Structures. IEEE TVCG, 18(4):651-659, 2012.

[16] P. Mohr, B. Kerbl, M. Donoser, D. Schmalstieg, and D. Kalkofen. Retargeting technical documentation to augmented reality. In Proc. ACM CHI, pages 3337-3346, 2015.

[17] O. Oda, C. Elvezio, M. Sukan, S. Feiner, and B. Tversky. Virtual Replicas for Remote Assistance in Virtual and Augmented Reality. In Proc. ACM UIST, pages 405-415, New York, NY, USA, 2015.

[18] R Core Team. R: A Language and Environment for Statistical Computing. R Foundation for Statistical Computing, 2015.

[19] C. M. Robertson, B. MacIntyre, and B. N. Walker. An evaluation of graphical context as a means for ameliorating the effects of registration error. IEEE TVCG, 15(2):179-192, 2009.

[20] R. Schmidt, K. Singh, and R. Balakrishnan. Sketching and Composing Widgets for 3D Manipulation. Computer Graphics Forum, 27(2):301-310, 2008.

[21] R. N. Shepard and J. Metzler. Mental Rotation of Three-Dimensional Objects. Science, 171(3972):701-703, 1971.

[22] R. Sodhi, H. Benko, and A. Wilson. Lightguide: projected visualizations for hand movement guidance. In Proc. ACM CHI, pages 179-188, 2012.

[23] S. G. Vandenberg and A. R. Kuse. Mental rotations, a group test of three-dimensional spatial visualization. Perceptual and motor skills, 47(2):599-604, 1978.

[24] D. Voyer, S. Voyer, and M. P. Bryden. Magnitude of sex differences in spatial abilities: a meta-analysis and consideration of critical variables. Psychological Bulletin, 117(2):250-270, 1995.

[25] M. Wexler, S. M. Kosslyn, and A. Berthoz. Motor processes in mental rotation. Cognition, 68(1):77-94, 1998.

[26] R. Whelan. Effective analysis of reaction time data. The Psychological Record, 58(3):475, 2008.

[27] S. White, L. Lister, and S. Feiner. Visual Hints for Tangible Gestures in Augmented Reality. In Proc. IEEE ISMAR, pages 47-50, 2007.

[28] A. Wohlschläger and A. Wohlschläger. Mental and manual rotation. Journal of Experimental Psychology: Human Perception and Performance, 24(2):397, 1998. 